\title{
The theory of young cluster disruption
}

\author{
Simon P. Goodwin
}

Dept. of Physics \& Astronomy, University of Sheffield, Hounsfield Road, Sheffield, S3 7RH, UK email: s.goodwin@sheffield.ac.uk

\begin{abstract}
Most stars seem to form in clusters, but the vast majority of these clusters do not seem to survive much beyond their embedded phase. The most favoured mechanism for the early destruction of star clusters is the effect of the removal of residual gas by feedback which dramatically changes the cluster potential. The effects of feedback depend on the ratio of the masses of stars and gas, and the velocity dispersion of the stars at the onset of gas removal. As gas removal is delayed by a few Myr from star formation these crucial parameters can change significantly from their initial values. In particular, in dynamically cool and clumpy clusters, the stars will collapse to a far denser state and if they decouple from the gas then gas removal may be far less destructive than previously thought. This might well help explain the survival of very massive clusters, such as globular clusters, without the need for extremely high star formation efficiencies or initial masses far greater than their current masses.
\end{abstract}

Keywords. clusters: general, stars: formation

\section{Introduction}

In our own Galaxy most star formation appears to occur in clusters (see reviews by Lada \& Lada 2003; Allen et al. 2007; Lada 2010), and in external galaxies most (at least high-mass) star formation is also observed in clusters (de Grijs 2010; Larsen 2010).

Observations of star formation and young clusters are relatively complete out to around $2 \mathrm{kpc}$ and find that clusters appear to form with a power-law initial cluster mass function $N(M) \propto M^{-\alpha}$ where $\alpha=1.7-2$ (Lada 2010). An interesting feature of an initial cluster mass function of this form is that all masses of clusters are equally important for populating the Galactic field. Low-mass clusters are far more numerous than high-mass clusters (e.g. Porras et al. 2003), but contribute the same total number of stars to the Galactic field (see e.g. Goodwin 2010).

When the numbers of clusters within a particular age range are examined however, it is found that there are far fewer old clusters than would be expected for a constant cluster formation rate. In particular, there are around ten times more embedded clusters than young (naked) open clusters in the Galaxy (Lada \& Lada 2003). This suggests that some form of 'infant mortality' it at work, destroying many clusters at very young $(<10 \mathrm{Myr})$ ages.

In this contribution I would like to discuss the theory of young cluster disruption: what might cause infant mortality, and what parameters of some young clusters might affect their survival and/or destruction.

\section{Gas expulsion}

Stars form from molecular gas, and the efficiency with which gas is turned into stars (the star formation efficiency, SFE) is often fairly low. The SFEs observed in clusterforming clumps in GMCs tend to be around 30\% (Lada \& Lada 2003). Therefore the 
mass of embedded clusters is mostly gas and this gas is removed after a few Myr by feedback from the most massive stars in a phase of 'gas removal'.

During gas removal the potential of the cluster will change drastically. Prior to gas removal the stars will be moving in a potential dominated by the gas, but the potential felt by the stars will reduce significantly, and often very rapidly, during gas removal. The effects of gas removal have been studied in detail by many authors both analytically and with the use of simulations (Tutukov 1978; Hills 1980; Mathieu 1983; Elmegreen 1983; Lada et al. 1984; Elmegreen \& Clemens 1985; Pinto 1987; Verschueren \& David 1989; Goodwin 1997a,b; Gyer \& Burkert 2001; Boily \& Kroupa 2003a,b; Bastian \& Goodwin 2006; Goodwin \& Bastian 2006; Baumgardt \& Kroupa 2007; Parmentier et al. 2008; Goodwin 2009; Chen \& Ko 2009).

It is often stated that the key parameter that controls the destructiveness of gas removal on a cluster is the SFE, modified by the timescale of gas removal. The lower the SFE the greater the contribution of gas to the potential and the more destructive gas removal will be (typically a figure of an SFE of $\sim 30 \%$ is given for a cluster to survive instantaneous gas removal). In addition, the slower the gas removal timescale, the less disruptive it is as the stars have a chance to adapt to the changing potential (lowering the critical SFE from $\sim 30 \%$ to $\sim 20 \%$ ).

Observed SFEs in cluster-forming clumps (of around $10^{3} M_{\odot}$ ) are observed to be of the same level as the theoretical minimum for cluster survival (Lada \& Lada 2003), therefore it might be expected that a lucky few clusters with a high-enough SFE can survive gas expulsion. However, the critical SFEs quoted for cluster survival are for the survival of a bound core which can be significantly less massive than the original cluster ('infant weightloss'). If the SFE is close to the critical SFE then $90 \%$ of the cluster mass may be lost with only a small bound cluster remaining (see Baumgardt \& Kroupa 2007). Therefore in the surviving cluster population (open and globulars) either these clusters were significantly more massive at birth (especially when considering evaporation from very old clusters), or they had extremely high SFEs.

However, there are two critical assumption that are generally made. Firstly, that the cluster is relatively smooth and spherical (most often it is modelled as a Plummer sphere). Secondly, that the cluster is in virial equilibriumt. It is not clear that either of these assumptions are correct.

\section{The initial conditions of clusters}

Observations strongly suggest that the stars in clusters form highly out-of-equilibrium with both non-virial velocity dispersions and large amounts of substructure (see for example Elmegreen \& Elmegreen 2001; Bertout \& Genova 2006; Allen et al. 2007; Kraus \& Hillenbrand 2008; Allison et al. 2009; Gutermuth et al. 2009; Clarke 2010 and references in all of these papers). This should not be surprising as in the gravoturbulent model of star formation stars will form in dense gas in filaments and clumps in a turbulent environment (see e.g. Elmegreen 2004; McKee \& Ostriker 2007; Bergin \& Tafalla 2007; Clarke 2010).

If a cluster is born substructured and sub-virial as observations and theory suggest that they often are (see above) then it will collapse in an attempt to reach a new equilibrium. However, initially a cluster will contain both collisionless stars and collisional gas so it is possible that the gas and stars may decouple, the stars collapsing to the centre of the

$\dagger$ A number of authors do consider non-virialised initial conditions such as Lada et al. (1984), Elmegreen \& Clemens (1985), and Verschueren \& David (1989). 
local potential well whilst the gas remains supported (although some, or a significant amount of gas may also fall into the potential well, see e.g. Maschberger et al. 2010; Moeckel \& Bate 2010).

In this process the stellar distribution will be approaching virial equilibrium, but the size of the stellar distribution will decrease (possibly very significantly) and the mass of gas in the same volume of the stars might well change (again, possibly significantly).

The effects of gas expulsion depend on the gas mass associated with the stars, and the velocity dispersion of the stars at the onset of gas expulsion - not at the time of formation. Therefore, there are two crucial parameters that evolve with time that determine the destructive effects of gas expulsion. Firstly, the effective SFE (eSFE, see Verschueren \& David 1989; Goodwin \& Bastian 2006; Goodwin 2009) which is the ratio of the stellar mass to gas mass at the onset of gas expulsion. Secondly, the velocity dispersion of the stars at the onset of gas expulsion (or how close to virial equilibrium the stars are with the combined gas and star potential, see Goodwin 2009).

Without large suites of full hydrodynamical simulations including feedback to correctly and self-consistently expel the gas it is unclear what exactly happens within clusters and how the effects of gas expulsion vary with the initial conditions of star formation (but see Allison et al. 2009, 2010; Maschberger et al. 2010; Moeckel \& Bate 2010).

\section{Conclusion}

To conclude. To form extremely massive clusters, such as globular clusters, it is not required to have either extremely high SFEs or

masses far greater at birth than now in order to overcome infant mortality and weightloss. Rather, if they are born in such as way that the effective SFE is low at the onset of gas expulsion then they will survive and lose little of their initial mass.

\section{References}

Allen, L., Megeath, S. T., Gutermuth, R., Myers, P. C., Wolk, S., Adams, F. C., Muzerolle, J., Young, E., \& Pipher, J. L. 2007, in 'Protostars and Planets V' eds. Reipurth, B., Jewitt, D. \& Keil, K. (University of Arizona Press: Tuscon), p 361

Allison, R. J., Goodwin, S. P., Parker, R. J., de Grijs, R., Portegies Zwart, S. F., \& Kouwenhoven, M. B. N. 2009, ApJ, 700, L99

Allison, R. J., Goodwin, S. P., Parker, R. J., Portegies Zwart, S. F., \& de Grijs, R. 2010, MNRAS, in press (arXiv:1004.5244)

Bastian, N. \& Goodwin, S. P. 2006, MNRAS, 369, L9

Baumgardt, H. \& Kroupa, P. 2007, MNRAS, 380, 1589

Bergin, E. \& Tafalla, M. 2007, ARAA, 45, 339

Bertout, C. \& Genova, F. 2006; $A \mathscr{E} A, 460,499$

Boily, C. M. \& Kroupa, P. 2003a, MNRAS, 338, 643

Boily, C. M. \& Kroupa, P. 2003a, MNRAS, 338, 673

Chen, H. \& Ko, C. 2009, ApJ, 698, 1659

Clarke, C. J. 2010, RSPTA, 368, 733

Elmegreen, B. G. 1983, MNRAS, 203, 1011

Elmegreen, B. G. \& Clemens, C. 1985, ApJ, 294, 523

Elmegreen, B. G. \& Elmegreen, D. M. 2001, AJ, 121, 1507

Elmegreen, B. G. 2004, ARAA, 42, 211

de Grijs, R. 2010, RSPTA, 368, 693

Goodwin, S. P. 1997a, MNRAS, 284, 785

Goodwin, S. P. 1997b, MNRAS, 286, 669

Goodwin, S. P. \& Bastian, N. 2006, MNRAS, 373, 752 
Goodwin, S. P. 2009, Ap\&SSS, 324, 259

Goodwin, S. P. 2010, RSPTA, 368, 851

Gutermuth, R. A., Megeath, S. T., Myers, P. C., Allen, L. E., Pipher, J. L. \& Fazio, G. G. 2009, ApJS, 184, 18

Gyer, M. P. \& Burkert, A. 2001, MNRAS, 323, 988

Hills, J. G. 1980, ApJ, 235, 986

Kraus, A. \& Hillenbrand, L. 2008, ApJ, 686, L111

Lada, C. J., Margulis, M. \& Dearborn, D. 1984, ApJ, 285, 141

Lada, C. J. \& Lada E. A. 2003, ARAA, 41, 57

Lada, C. J. 2010, RSPTA, 368, 713

Larsen, S. S. 2010, RSPTA, 368, 867

McKee, C. \& Ostriker, E. 2007, ARAA, 45, 565

Maschberger, Th., Clarke, C. J., Bonnell, I. A. \& Kroupa, P. 2010, MNRAS, 404, 1061

Mathieu, R. D. 1983, ApJ, 267, 97

Moeckel, N. \& Bate, M. R. 2010, MNRAS, 404, 721

Parmentier, G., Goodwin, S. P., Kroupa, P. \& Baumgardt, H. 2008, ApJ, 678, 347

Pinto, F. 1987, PASP, 99, 1161

Porras, A., Christopher, M., Allen, L., Di Francesco, J., Megeath, S. T., \& Myers, P. C. 2003, ApJ, 126, 1916

Tutukov, A. V. 1978, A\& A, 70, 57

Verschueren, W. \& David, M. 1989, A\& A, 219, 105 